\title{
LACUNARY STATISTICAL CONVERGENCE OF MULTIPLE SEQUENCES IN INTUITIONISTIC FUZZY NORMED SPACES
}

\author{
Ekrem Savaş \\ Department of Mathematics, Istanbul Ticaret University, Üsküdar, Istanbul, Turkey \\ ekremsavas@yahoo.com
}

\begin{abstract}
In this paper we introduce the notion ideal statistical convergence and ideal lacunary statistical convergence of multiple sequences with respect to the intuitionistic fuzzy norm $(\mu, v)$, investigate their relationships, and make some observations about these classes.

Key Words- Ideal, Filter, $I$-double statistical convergence, $I$-double lacunary statistical convergence, multiple sequences, double lacunary statistical convergence, closed subspace.
\end{abstract}

\section{INTRODUCTION}

The notion of statistical convergence is a very useful functional tool for studying the convergence problems of numerical sequences/matrices (double sequences) through the concept of density. It was first introduced by Fast [1], and Schoenberg [2], independently for the real sequences. Later on it was further investigated from sequence point of view and linked with the summability theory by Fridy [3], Salat [4] and many others. The idea is based on the notion of natural density of subsets of $\square$, the set of positive integers, which is defined as follows: The natural density of a subset $K$ of $\square$ is denoted by $\delta(K)$ and is defined by

$$
\delta(K)=\lim _{n \rightarrow \infty} \frac{1}{n}|\{k \in K: k \leq n\}|,
$$

where the vertical bar denotes the cardinality of the respective set. ( see, [5]).

In another direction, a new type of convergence, called lacunary statistical convergence, was introduced in [6] as follows. A lacunary sequence is an increasing integer sequence $\theta=\left\{k_{r}\right\}_{r \in \square \cup\{0\}}$ such that $k_{0}=0$ and $h_{r}=k_{r}-k_{r-1} \rightarrow \infty$, as $r \rightarrow \infty$. Let $I_{r}=\left(k_{r-1}, k_{r}\right]$ and $q_{r}=\frac{k_{r}}{k_{r-1}}$.

A sequence $\left(x_{k}\right)$ of real numbers is said to be lacunary statistically convergent to $L$ (or $S_{\theta}$-convergent to $L$ ) if, for any $\varepsilon>0$,

$$
\lim _{r \rightarrow \infty} \frac{1}{h_{r}}\left|\left\{k \in I_{r}:\left|x_{k}-L\right| \geq \varepsilon\right\}\right|=0
$$

where $|A|$ denotes the cardinality of $A \subset \square$. In [6], the relation between lacunary statistical convergence and statistical convergence was established, among other things. Recently Savas and Patterson [7] introduced and studied lacunary statistical convergence for double sequences and also some inclusion theorems are presented for 
real sequences. Recently, Mohiuddine and Aiyub [8] studied lacunary statistical convergence as generalization of the statistical convergence introduced the concept $\theta$ statistical convergence in random 2-normed space. In [9], Mursaleen and Mohiuddine extended the idea of lacunary statistical convergence with respect to the intuitionistic fuzzy normed space. Also lacunary statistically convergent double sequences in probabilistic normed space was studied by Mohiuddine and Savaş in [10]. Maio and Kocinac [11] introduced statistical convergence in topology. In [12], some new double sequence spaces of fuzzy real numbers by combining I-convergence, Orlicz function and four dimensional matrix are introduced. More results on this convergence can be found in $[13,14,15,16,17,18]$.

The concepts of fuzzy sets and fuzzy set operations were first introduced by Zadeh [19]. Subsequently several authors have discussed various aspects of the theory and applications of fuzzy sets. The theory of intuitionistic fuzzy sets was introduced by Atanassov [20]; it has been extensively used in decision-making problems [21]. The concept of an intuitionistic fuzzy metric space was introduced by Park [22]. Further, Saadati and Park [23] gave the notion of an intuitionistic fuzzy normed space. Some works related to the convergence of sequences in several normed linear spaces in a fuzzy setting can be found in [24, 25, 26, 27, 28, 29].

In this paper, as a variant of double statistical convergence, the notions of ideal double statistical convergence and ideal lacunary double statistical convergence are introduced in an intuitionistic fuzzy normed linear space, which naturally extend the notions of double statistical convergence and double lacunary statistical convergence and some important results are established. Furthermore, we try to establish the relations between these two summability notions.

Throughout the paper, $\square$ will denote the set of all natural numbers. First we shall give some basic definitions used in this paper.

Definition 1.1. ([30]) A triangular norm (t-norm) is a continuous mapping $*:[0,1] \times[0,1] \rightarrow[0,1]$ such that $(\mathrm{S}, *)$ is an abelian monoid with unit one and $c * d \leq a * b$ if $c \leq a$ and $d \leq b$ for all $a, b, c, d \in[0,1]$.

Definition 1.2. ([30]) A binary operation $\diamond:[0,1] \times[0,1] \rightarrow[0,1]]$ is said to be a continuous t-conorm if it satisfies the following conditions:

(i) $\diamond$ is associate and commutative,

(ii) $\diamond$ is continuous,

(iii) $a \diamond 0=a$ for all $a \in[0,1]$,

(iv) $a \diamond b \leq c \diamond d$ whenever $a \leq c$ and $b \leq d$ for each $a, b, c, d \in[0,1]$.

For example, we can give $a * b=a b, a * b=\min \{a, b\}, a \diamond b=\min \{a+b, 1\}$

and $a \diamond b=\max \{a, b\}$ for all $a, b \in[0,1]$.

Using the continuous $t$-norm and $t$-conorm, Saadati and Park [23] has recently introduced the concept of intuitionistic fuzzy normed space as follows. 
Definition1.3. ([23]) The 5-tuple $(X, \mu, v, *, \diamond)$ is said to be an intuitionistic fuzzy normed space (for short, IFNS) if $X$ is a vector space, $*$ is a continuous $t$-norm, $\diamond$ is a continuous $t$-conorm, and $\mu, v$ are fuzzy sets on $X \times(0, \infty)$ satisfying the following conditions for every $x, y \in X$, and $s, t>0$ :

a) $\mu(x, t)+v(x, t) \leq 1$,

b) $\mu(x, t)>0$,

c) $\mu(x, t)=1$ if and only if $x=0$,

d) $\mu(\alpha x, t)=\mu\left(x, \frac{t}{|\alpha|}\right)$ for each $\alpha \neq 0$,

e) $\mu(x, t) * \mu(y, s) \leq \mu(x+y, t+s)$,

f) $\mu(x,):.(0, \infty) \rightarrow[0,1]$ is continuous,

g) $\lim _{t \rightarrow \infty} \mu(x, t)=1$ and $\lim _{t \rightarrow 0} \mu(x, t)=0$,

h) $v(x, t)<1$,

i) $v(x, t)=0$ if and only if $x=0$,

j) $v(\alpha x, t)=\mu\left(x, \frac{t}{|\alpha|}\right)$ for each $\alpha \neq 0$,

k) $v(x, t) \diamond v(y, s) \geq v(x+y, t+s)$,

l) $v(x,):.(0, \infty) \rightarrow[0,1]$ is continuous,

m) $\lim _{t \rightarrow \infty} v(x, t)=0$ and $\lim _{t \rightarrow 0} v(x, t)=1$.

In this case $(\mu, v)$ is called an intuitionistic fuzzy norm. As a standard example, we can give the following:

Let $(X, \square . \square)$ be a normed space, and let $a * b=a b$ and $a \diamond b=\min \{a+b, 1\}$ for all $a, b \in[0,1]$. For all $x \in X$ and every $t>0$, consider

$\mu(x, t)=\frac{t}{t+\square x \square}$ and $v_{0}(x, t)=\frac{\square x \square}{t+\square x \square}$.

Then observe that $(X, \mu, v, *, \diamond)$ is an intuitionistic fuzzy normed space.

We also recall that the concept of double convergence in an intuitionistic fuzzy normed space is studied in [31].

Definition 1.4. ([31]) Let $(X, \mu, \mathrm{v}, *, \diamond)$ be an IFNS. Then, a sequence $x=\left(x_{k, l}\right)$ is said to be convergent to $L \in X$ with respect to the intuitionistic fuzzy norm $(\mu, v)$ if, for every $\varepsilon>0$ and $t>0$, there exists $\left(k_{0}, l_{0}\right) \in \square \times \square$ such that $\mu\left(x_{k, l}-L, t\right)>1-\varepsilon$ 
and $v\left(x_{k, l}-L, t\right)<\varepsilon$ for all $k \geq k_{0}$ and $l \geq l_{0}$. It is denoted by $(\mu, v)_{2}-\lim x=L$ or $x_{k, l} \stackrel{(\mu, v)_{2}}{\rightarrow} L$ as $k, l \rightarrow \infty$.

\section{I- DOUBLE STATISTICAL AND I- DOUBLE LACUNARY STATISTICAL CONVERGENCE ON IFNS}

In this section we deal with the relation between these two new methods as also the relation between $I_{\theta}$-double statistical convergence and $I$-double statistical convergence in an intuitionistic fuzzy normed space $(\mu, v)$. Before proceeding further, we should recall some notation on the $I$-double statistical convergence and ideal convergence.

Statistical convergence of double sequences $x=\left(x_{k, l}\right)$ has been defined and studied by Mursaleen and Edely [32]; and for fuzzy numbers by Savas and Mursaleen [33].

Let $K \subseteq \square \times \square$ be a two-dimensional set of positive integers and let $K(m, n)$ be the numbers of $(k, l)$ in $K$ such that $k \leq m$ and $l \leq n$. Then the two-dimensional analogue of natural density can be defined as follows [32].

The lower asymptotic density of the set $K \subseteq \square \times \square$ is defined as

$$
\underline{\delta_{2}}(K)=\liminf _{m ; n} \frac{K(m, n)}{m n} \text {. }
$$

In case the sequence $(K(m, n) / m n)$ has a limit then we say that $K$ has a double natural density and is defined as

$$
\lim _{m ; n} \frac{K(m, n)}{m n}=\delta_{2}(K) \text {. }
$$

Definition 2.1. ([32]). A real double sequence $x=\left(x_{k, l}\right)$ is said to be statistically convergent to the number $\mathrm{L}$ if for each $\varepsilon>0$, the set

$$
\left\{(k, l), k \leq m \text { and } l \leq n:\left|x_{k, l}-L\right| \geq \varepsilon\right\}
$$

has double natural density zero. In this case we write $s t_{2}-\lim _{k l} x_{k, l}=L$.

P. Kostyrko et al [33] and [34] introduced the concept of $I$ - convergence of sequences in a metric space and studied some properties of such convergence. Note that $I$ convergence is an interesting generalization of statistical convergence. More investigations in this direction and more applications of ideals can be found in $[36,37$, 38, 39, 40, 41 ].

Before proceeding further, we should recall some notation on the ideal.

A family $I \subset 2^{Y}$ of subsets of a nonempty set $Y$ is said to be an ideal in $Y$ if

i) $\varnothing \in I$;

ii) $A, B \in I$ imply $A \cup B \in I$;

iii) $A \in I, B \subset A$ imply $B \in I$. 
A proper ideal $I$ is said to be admissible if $\{n\} \in I$ for each $n \in \square$.

i) $\varnothing \notin F$;

ii) $A, B \in F$ imply $A \cap B \in F$;

iii) $A \in F, A \subset B$ imply $B \in F$.

If $I$ is a proper ideal of $\square \quad$ (i.e., $\square \notin I$ ), then the family of sets $F(I)=\{M \subset N: \exists A \in I: M=\square \backslash A\}$ is a filter of $\square$. It is called the filter associated with the ideal.

Throughout, $I$ will stand for a proper admissible ideal of $\square$.

Definition 2.2. A sequence $x=\left(x_{k, l}\right)$ is said to be $I$-double statistically convergent sequences to $L$ or $S(I)_{2}$-convergent to $L$ if for each $\varepsilon>0$ and $\delta>0$

$\left\{(m, n) \in \square \times \square: \frac{1}{(m n)} \mid\left\{k \leq m\right.\right.$ and $\left.\left.l \leq n:\left|x_{k, l}-L\right| \geq \varepsilon\right\} \mid \geq \delta\right\} \in I$,

or equivalently if for each $\varepsilon>0$

$\delta_{\mathrm{I}}^{2}(\mathrm{~A}(\varepsilon))=I-\lim \delta_{m n}(\mathrm{~A}(\varepsilon))=0$

where $A(\varepsilon)=\left\{k \leq m\right.$ and $\left.l \leq n:\left|x_{k, l}-L\right| \geq \varepsilon\right\}$ and $\delta_{m n}(A(\varepsilon))=\frac{|A(\varepsilon)|}{(m n)}$.

In this case we write $x_{k, l} \rightarrow L\left(S(I)_{2}\right)$. The class of all $I$ - double statistically convergent sequences will be denoted by simply $S(I)_{2}$.

Remark 1. For $I=I_{\text {fin }}, S(I)_{2}$-convergence coincides with double statistical convergence, [32].

Definition 2.3. [25]. Let $(X, \mu, v, *, \diamond)$ be an IFNS. Then, a sequence $x=\left(x_{k, l}\right)$ is said to be $I$ - double statistically convergent sequences to $L$ with respect to the intuitionistic fuzzy normed space $(\mu, v)$, if for every $\varepsilon>0$, and every $\delta>0$ and $t>0$,

$\left\{(m, n) \in \square \times \square: \frac{1}{(m n)} \mid\left\{k \leq m\right.\right.$ and $l \leq n: \mu\left(x_{k, l}-L, t\right) \leq 1-\varepsilon$ or $\left.\left.v\left(x_{k, l}-L, t\right) \geq \varepsilon\right\} \mid \geq \delta\right\} \in I$.

In this case we write $x_{k, l} \stackrel{(\mu, v)}{\rightarrow} L\left(S^{(\mu, v)}(I)_{2}\right)$. The class of all $I$ - double statistically convergent sequences to $L$ with respect to the intuitionistic fuzzy normed space $(\mu, v)$ will be denoted simply by $S^{(\mu, v)}(I)_{2}$.

Remark 2. For $I=I_{f i n}, S^{(\mu, v)}(I)_{2}$-convergence coincides with double statistical convergence with respect to the intuitionistic fuzzy normed space $(\mu, v),[31]$. 
Definition 2.4. The double sequence $\theta_{r ; s}=\left\{\left(k_{r}, l_{s}\right)\right\}$ is called double lacunary if there exist two increasing of integers such that

$k_{0}=0, h_{r}=k_{r}-k_{r-1} \rightarrow \infty$ as $r \rightarrow \infty$

and

$l_{0}=0, \bar{h}_{s}=l_{s}-l_{s-1} \rightarrow \infty$ as $s \rightarrow \infty$.

Notations: $k_{r, s}=k_{r} l_{s}, h_{r, s}=h_{r} \bar{h}_{s}, \theta_{r, s}$ is determine by

$I_{r, s}=\left\{(k, l): k_{r-1}<k \leq k_{r} \& l_{s-1}<l \leq l_{s}\right\}, \quad q_{r}=k_{r} k_{r-1}, q_{s}=l_{s} l_{s-1}$, and $q_{r, s}=q_{r} q_{s}$. We will denote the set of all double lacunary sequences by $\mathrm{N}_{\theta_{\mathrm{r} ; s}}$.

We now ready to obtain our main definitions and results.

Definition 2.5. Let $(X, \mu, v, *, \diamond)$ be an IFNS and $\theta$ be a double lacunary sequence. A sequence $x=\left(x_{k, l}\right)$ is said to be $I$ - double lacunary statistically convergent to $L \in X$ with respect to the intuitionistic fuzzy norm $(\mu, v)$ if, for any $\varepsilon>0, t>0$ and $\delta>0$, $\left\{(r, s) \in \square \times \square: \frac{1}{h r s} \mid\left\{(k, l) \in I_{r s}: \mu\left(x_{k, l}-L, t\right) \leq 1-\varepsilon\right.\right.$ or $\left.\left.v\left(x_{k, l}-L, t\right) \geq \varepsilon\right\} \mid \geq \delta\right\} \in I$.

In this case, we write $x_{k, l} \stackrel{(\mu, v)}{\rightarrow} L\left(S_{\theta}^{(\mu, v)}(I)_{2}\right)$. The class of all $I$ - lacunary statistically convergent sequences will be denoted by $S_{\theta}^{(\mu, v)}(I)_{2}$.

It can be checked as in the case of $I$-double statistically and $I$-double lacunary statistically convergent sequences with respect to the intuitionistic fuzzy normed space $(\mu, v)$ that both $S^{(\mu, v)}(I)_{2}$ and $S_{\theta}^{(\mu, v)}(I)_{2}$ are linear subspaces of the space of real sequences.

As the proofs for both the assertions are similar, we present the proof for $S_{\theta}^{(\mu, v)}(I)_{2}$ only.

Theorem 2.1. $S_{\theta}^{(\mu, v)}(I)_{2} \cap l_{\infty}^{(\mu, v)_{2}}$ is a closed subset of $l_{\infty}^{(\mu, v)_{2}}$, where $l_{\infty}^{(\mu, v)_{2}}$ stands for the space of all double bounded sequences of intuitionistic fuzzy norm $(\mu, v)$.

Proof. Assume that $\left(x^{m n}\right) \subset S_{\theta}^{(\mu, v)}(I)_{2} \cap \ell_{\infty}^{(\mu, v)_{2}}$, is a convergent sequence and it converges to $x \in \ell_{\infty}^{(\mu, v)_{2}}$. We need to show that $x \in S_{\theta}^{(\mu, v)}(I)_{2} \cap \ell_{\infty}^{(\mu, v)_{2}}$. Suppose that $x^{m n} \rightarrow L_{m n}\left(S_{\theta}^{(\mu, v)}(I)_{2}\right)$ for all $(m, n) \in \square \times \square$. Take a positive strictly decreasing sequence $\left\{\varepsilon_{m n}\right\}$ where $\varepsilon_{m n}=\frac{\varepsilon}{2^{m n}}$ for a given $\varepsilon>0$. Clearly $\left\{\varepsilon_{m n}\right\}$ converges to 0 . Choose a positive integer $(m, n)$ such that $\left\|x-x^{m n}\right\|_{\infty}=\sup _{m n}\left\{v\left(x-x^{m n}, t\right)\right\}<\frac{\varepsilon_{m n}}{4}$. Let $0<\delta<1$. Then 
$A_{\mu, v}\left(\varepsilon_{m n}, t\right)=\left\{(r, s) \in \square \times \square: \frac{1}{h_{r s}}\left|\left\{\begin{array}{c}(k, l) \in I_{r s}: \mu\left(x_{k, l}^{m n}-L_{m n}, t\right) \leq 1-\frac{\varepsilon_{m n}}{4} \text { or } \\ v\left(x_{k, l}^{m n}-L_{m n}, t\right) \geq \frac{\varepsilon_{m n}}{4}\end{array}\right\}\right|<\frac{\delta}{3}\right\} \in F(I)$ and

$$
\begin{gathered}
B_{\mu v}\left(\varepsilon_{m n}, t\right)=\{(r, s) \in \square \times \square: \\
\frac{1}{h_{r s}}\left|\left\{\begin{array}{l}
(k, l) \in I_{r s}: \mu\left(x_{k, l}^{m+1, n+1}-L_{m+1, n+1}, t\right) \leq 1-\frac{\varepsilon_{m+1, n+1}}{4} \text { or } \\
v\left(x_{k, l}^{m+1, n+1}-L_{m+1, n+1}, t\right) \geq \frac{\varepsilon_{m+1, n+1}}{4}
\end{array}\right\}\right| \leq \frac{\delta}{3} \in F(I) .
\end{gathered}
$$

Since $\quad A_{\mu, v}\left(\varepsilon_{m n}, t\right) \bigcap B_{\mu, v}\left(\varepsilon_{m n}, t\right) \in F(I) \quad$ and $\quad \varnothing \notin F(I)$, we can choose $(r, s) \in A_{\mu, v}\left(\varepsilon_{m n}, t\right) \cap B_{\mu, v}\left(\varepsilon_{m n}, t\right)$. Then

$$
\frac{1}{h_{r s}} \mid\left\{(k, l) \in I_{r s}: \mu\left(x_{k, l}^{m+1, n+1}-L_{m+1, n+1}, t\right) \leq 1-\frac{\varepsilon_{m+1, n+1}}{4} \text { or } v\left(x_{k, l}^{m+1, n+1}-L_{m+1, n+1}, t\right) \geq \frac{\varepsilon_{m+1, n+1}}{4}\right\} \mid<\frac{\delta}{3} \text {. }
$$

Hence, there exist $\quad(k, l) \in I_{r s} \quad$ for $\quad$ which $\quad \mu\left(x_{k, l}^{m+1, n+1}-L_{m+1, n+1}, t\right)>1-\frac{\varepsilon_{m n}}{4} \quad$ or $v\left(x_{k, l}^{m n}-L_{m . n}, t\right)<\frac{\varepsilon_{m n}}{4} \quad$ and $\quad \mu\left(x_{k, l}^{m+1, n+1}-L_{m+1, n+1}, t\right)>1-\frac{\varepsilon_{m+1, n+1}}{4} \quad$ or $v\left(x_{k, l}^{m+1, n+1}-L_{m+1, n+1}, t\right)<\frac{\varepsilon_{m+1, n+1}}{4}$.

Then .we can write

$$
\begin{aligned}
v\left(L_{m, n}, L_{m+1, n+1}, t\right) & \leq v\left(L_{m n}-x_{k, l}^{m n}, \frac{t}{3}\right) \diamond v\left(x_{k, l}^{m n}-x_{k, l}^{m+1, n+1}, \frac{t}{3}\right) \diamond v\left(x_{k, l}^{m+1, n+1}-L_{m+1, n+1}, \frac{t}{3}\right) \\
& \leq v\left(x_{k, l}^{m n}-L_{m, n}, \frac{t}{3}\right) \diamond v\left(x_{k, l}^{m+1, n+1}-L_{m+1, n+1}, \frac{t}{3}\right) \oslash \sup _{m n}\left\{v\left(x-x^{m n}, t\right)\right\} \sup _{m n}\left\{\left(x-x^{m+1, n+1}, t\right)\right\} \\
& \leq \frac{\varepsilon_{m n}}{4} \oslash \frac{\varepsilon_{m+1, n+1}}{4} \oslash \frac{\varepsilon_{m n}}{4} \oslash \frac{\varepsilon_{m+1, n+1}}{4} \leq \varepsilon_{m n} .
\end{aligned}
$$

This implies that $\left\{L_{m n}\right\}$ is a Cauchy sequence in $\square$, and so there is a real number $L$ such that $L_{m n} \rightarrow L$, as $m n \rightarrow \infty$. We need to prove that $x \rightarrow L\left(S^{(\mu, v) \theta}(I)_{2}\right)$. For any $\varepsilon>0$ and $t>0$, choose $(m, n) \in \square \times \square$ such that $\varepsilon_{m n}<\frac{\varepsilon}{4},\left\|x-x^{m n}\right\|_{\infty}=\sup _{m n}\left\{v\left(x-x^{m n}, t\right)\right\}<\frac{\varepsilon}{4}$,

$$
\begin{gathered}
\mu\left(L_{m n}-L, t\right)<1-\frac{\varepsilon}{4} \text { or } v\left(L_{m n}-L, t\right)<\frac{\varepsilon}{4} \text {. Then } \\
\frac{1}{h_{r s}}\left|\left\{(k, l) \in I_{r s}: v\left(x_{k, l}-L, t\right) \geq \varepsilon\right\}\right| .
\end{gathered}
$$


This implies that

$$
\begin{aligned}
& \leq \frac{1}{h_{r s}} \mid\left\{\begin{array}{l}
(k, l) \in I_{r s}: v\left(x_{k, l}^{m, n}-L_{m, n}, t\right) \sup _{m n}\left\{v \left(x_{k l}-x_{k l}^{m n}, t\right.\right. \\
\diamond v\left(L_{m n}-L, t\right) \geq \varepsilon
\end{array}\right\} . \\
& \leq \frac{1}{h_{r s}}\left|\left\{(k, l) \in I_{r s}: v\left(x_{k, l}^{m, n}-L_{m, n}, t\right) \diamond \frac{\varepsilon}{4} \diamond \frac{\varepsilon}{4} \geq \varepsilon\right\}\right| . \\
& \leq \frac{1}{h_{r s}}\left|\left\{(k, l) \in I_{r s}: v\left(x_{k, l}^{m, n}-L_{m, n}, t\right) \geq \frac{\varepsilon}{2}\right\}\right| .
\end{aligned}
$$

$$
\begin{aligned}
& \left.\left\{(r, s) \in \square \times \square: \frac{1}{h_{r s}} \mid(k, l) \in I_{r s}: \mu\left(x_{k, l}-L, t\right) \leq 1-\operatorname{\varepsilon } \operatorname{orv}\left(x_{k, l}-L, t\right) \geq \varepsilon\right\} \mid<\delta\right\} \\
& \supseteq\left\{(r, s) \in \square \times \square: \frac{1}{h_{r s}}\left|\left\{(k, l) \in I_{r s}: \mu\left(x_{k, l}^{m n}-L_{m n}, t\right) \leq 1-\frac{\varepsilon}{2} \operatorname{orv}\left(x_{k, l}^{m n}-L_{m n}, t\right) \geq \frac{\varepsilon}{2}\right\}\right|<\delta\right\} \in F(I) .
\end{aligned}
$$

So

$$
\left.\left\{(r, s) \in \square \times \square: \frac{1}{h_{r s}} \mid(k, l) \in I_{r s}: \mu\left(x_{k, l}-L, t\right) \leq 1-\operatorname{\varepsilon orv}\left(x_{k, l}-L, t\right) \geq \varepsilon\right\} \mid<\delta\right\} \in F(I) .
$$

and so

$$
\left.\left\{(r, s) \in \square \times \square: \frac{1}{h_{r s}} \mid(k, l) \in I_{r s}: \mu\left(x_{k, l}-L, t\right) \leq 1-\varepsilon \text { or } v\left(x_{k, l}-L, t\right) \geq \varepsilon\right\} \mid<\delta\right\} \in I .
$$

This gives that $x_{k, l} \rightarrow L\left(S_{\theta}{ }^{(\mu, v)}(I)_{2}\right)$, and this completes the proof of the theorem.

Definition 2.6. Let $\theta$ be double lacunary sequence. Then $x=\left(x_{k, l}\right)$ is said to be $N_{\theta}^{(\mu, v)}(I)_{2}$-convergent to $L \in X$ with respect to the intuitionistic fuzzy norm $(\mu, v)$ if, for any $\varepsilon>0$ and $t>0$.,

$\left\{(r, s) \in \square \times \square: \frac{1}{h_{r s}} \sum_{(k, l) \in I_{r s}} \mu\left(x_{k, l}-L, t\right) \leq 1-\varepsilon\right.$ or $\left.\sum_{(k, l) \in I_{r s}} v\left(x_{k, l}-L, t\right) \geq \varepsilon\right\} \in I$.

This convergence is denoted by $x_{k l} \rightarrow L\left(N_{\theta}{ }^{(\mu, v)}(I)_{2}\right)$, and the class of such sequences will be denoted simply by $N_{\theta}^{(\mu, v)}(I)_{2}$.

Theorem 2.2. Let $\theta=\left(k_{r s}\right)$ be a lacunary sequence. Then i)(a) $x_{k, l} \rightarrow L\left(N_{\theta}^{(\mu, v)}(I)_{2}\right) \Rightarrow x_{k, l} \rightarrow L\left(S_{\theta}^{(\mu, v)}(I)_{2}\right)$, and

(b) $N_{\theta}^{(\mu, v)}(I)_{2}$ is a proper subset of $S_{\theta}^{(\mu, v)}(I)_{2}$.

ii) $x \in l_{\infty}^{(\mu, v)_{2}}$ and $x_{k, l} \rightarrow L\left(S_{\theta}^{(\mu, v)}(I)_{2}\right) \Rightarrow x_{k, l} \rightarrow L\left(N_{\theta}^{(\mu, v)}(I)_{2}\right)$,

iii) $S^{(\mu, v)_{\theta}}(I)_{2} \cap l_{\infty}^{(\mu, v)_{2}}=N_{\theta}^{(\mu, v)}(I)_{2} \cap l_{\infty}^{(\mu, v)_{2}}$.

Proof. i) (a) If $\varepsilon>0$ and $x_{k, l} \rightarrow L\left(N_{\theta}^{(\mu, v)}(I)_{2}\right)$, we can write 


$$
\begin{aligned}
& \sum_{(k, l) \in I_{r s}}\left(\mu\left(x_{k, l}-L, t\right) \text { or } v\left(x_{k, l}-L, t\right)\right) \\
& \geq \sum_{\substack{(k, l) \in I_{r s}, \mu\left(x_{k l}-L, t\right)<1-\varepsilon \\
\text { or } \mu\left(x_{k l}-L, t\right) \geq \varepsilon}}\left(\mu\left(x_{k, l}-L, t\right) \text { or } v\left(x_{k, l}-L, t\right)\right. \\
& \geq \varepsilon \mid\left\{(k, l) \in I_{r s}: \mu\left(x_{k, l}-L, t\right) \leq 1-\varepsilon \text { or } v\left(x_{k, l}-L, t\right) \geq \varepsilon\right\} \mid
\end{aligned}
$$

and so

$\frac{1}{\varepsilon h_{r s}} \sum_{(k, l) \in I_{r s}} \mu\left(x_{k, l}-L, t\right)$ or $v\left(x_{k, l}-L, t\right)$

$\geq \frac{1}{h_{r s}} \mid(k, l) \in I_{r s}: \mu\left(x_{k, l}-L, t\right) \leq 1-\varepsilon$ or $\varepsilon v\left(x_{k, l}-L, t\right) \geq \varepsilon \mid$.

Then, for any $\varepsilon>0$ and $t>0$.,

$\left\{(r, s) \in \square \times \square: \frac{1}{h_{r s}} \mid(k, l) \in I_{r s}: \mu\left(x_{k, l}-L, t\right) \leq 1-\varepsilon \quad\right.$ or $\left.\left.\quad v\left(x_{k l}-L, t\right) \geq \varepsilon\right\} \mid \geq \delta\right\}$.

$\subseteq\left\{(r, s) \in \square \times \square: \frac{1}{h_{r s}} \sum_{(k, l) \in I_{r s}} \mid \mu\left(x_{k l}-L, t\right) \leq(1-\varepsilon) \delta\right.$ or $\left.\left.\frac{1}{h_{r s}} \sum_{(k, l) \in I_{r s}} v\left(x_{k l}-L, t\right) \geq \varepsilon\right\} \mid<\varepsilon . \delta\right\} \in I$.

This proves the result.

(b) In order to establish that the inclusion $N_{\theta}^{(\mu, v)}(I)_{2} \subseteq S_{\theta}^{(\mu, v)}(I)_{2}$ is proper, let $\theta$ be given and let $x$ defined as follows:

$x_{k, l}:=\left(\begin{array}{ccccccc}1 & 2 & 3 & \cdots & {\left[\sqrt[3]{h_{r, s}}\right]} & 0 & \cdots \\ 2 & 2 & 3 & \cdots & {\left[\sqrt[3]{h_{r, s}}\right]} & 0 & \cdots \\ \vdots & \vdots & \vdots & \vdots & \vdots & \vdots & \vdots \\ 2 & {\left[\sqrt[3]{h_{r, s}}\right]} & \cdots & \cdots & {\left[\sqrt[3]{h_{r, s}}\right]} & 0 & \cdots \\ 0 & 0 & 0 & 0 & 0 & 0 & \vdots \\ \vdots & \vdots & \vdots & \vdots & \vdots & \vdots & \ddots\end{array}\right)$

Then, for any $\varepsilon>0$ and $t>0$

$\frac{1}{h_{r s}} \mid(k, l) \in I_{r s}: \mu\left(x_{k l}-0, t\right) \leq 1-\varepsilon$ or $\left.v\left(x_{k l}-0, t\right) \geq \varepsilon\right\} \mid \leq \frac{\left[\sqrt[3]{h_{r s}}\right]}{h_{r s}}$,

and for any $\delta>0$ we get

$\left\{(r, s) \in \square \times \square: \frac{1}{h_{r s}} \mid(k, l) \in I_{r s}: \mu\left(x_{k, l}-0, t\right) \leq 1-\varepsilon \quad\right.$ or $\left.\left.\quad v\left(x_{k, l}-0, t\right) \geq \varepsilon\right\} \mid \geq \delta\right\}$

$\subseteq\left\{(r, s) \in \square \times \square: \frac{\left[\sqrt[3]{h_{r s}}\right]}{h_{r s}} \geq \delta\right\}$.

Since the set on the right-hand side is a finite set and so belongs to $I$, it follows that $x_{k, l} \rightarrow O\left(S_{\theta}^{(\mu, v)}(I)_{2}\right)$.

On the other hand, 
$\frac{1}{h_{r s}} \sum_{(k, l) \in I_{r s}} \mu\left(x_{k, l}-0, t\right)$ or $\left.v\left(x_{k, l}-0, t\right)\right)=\frac{1}{h_{r s}} \cdot \frac{\left[\sqrt[3]{h_{r s}}\right]\left(\left[\sqrt[3]{h_{r s}}\right]\left(\left[\sqrt[3]{h_{r s}}\right]+1\right)\right)}{2}$.

Then

$$
\begin{aligned}
& \left\{(r, s) \in \square \times \square: \frac{1}{h_{r s}} \sum_{(k, l) \in I_{r s}} \mu\left(x_{k, l}-0, t\right) \leq 1-\frac{1}{4} \text { or } \frac{1}{h_{r s}} \sum_{(k, l) \in I_{r s}} v\left(x_{k, l}-0, t\right) \geq \frac{1}{4}\right\} \\
& =\left\{(r, s) \in \square \times \square: \frac{\left[\sqrt[3]{h_{r, s}}\right]\left(\left[\sqrt[3]{h_{r, s}}\right]\left(\left[\sqrt[3]{h_{r, s}}\right]+1\right)\right)}{h_{r s}} \geq \frac{1}{2}\right\}
\end{aligned}
$$

for some $(r, s) \in \square x \square$ which belongs to $F(\mathrm{I})$, since $I$ is admissible. So $x_{k, l} \nrightarrow 0\left(N_{\theta}^{(\mu, v)}(I)_{2}\right)$.

ii) Suppose that $x_{k, l} \rightarrow L\left(S_{\theta}^{(\mu, v)}(I)_{2}\right)$ and $x \in l_{\infty}^{(\mu, v)}$. Then there exists an $M>0$ such that $\mu\left(x_{k, l}-L, t\right) \leq 1-M$ or $v\left(x_{k, l}-L, t\right) \geq M \forall(k, l) \in \square \times \square$. Given $\varepsilon>0$, we have

$$
\begin{aligned}
& \frac{1}{h_{r s}} \sum_{(k, l) \in I_{r s}}\left(\mu\left(x_{k, l}-L, t\right) \text { or } v\left(x_{k, l}-L, t\right)\right) \\
& =\frac{1}{h_{r s}} \sum_{\substack{(k, l) \in I_{r s}, \mu\left(x_{k, l}-L, t\right) \leq 1-\frac{\varepsilon}{2} \\
\text { or } v\left(x_{k, l}-L, t\right) \geq \frac{\varepsilon}{2}}}\left(\mu\left(x_{k, l}-L, t\right) \text { or } v\left(x_{k, l}-L, t\right)\right) \\
& +\frac{1}{h_{r s}} \sum_{\substack{(k, l) \in I_{r s}, \mu\left(x_{k, l}-L, t\right)>1-\frac{\varepsilon}{2} \\
\text { or } v\left(x_{k, l}-L, t\right)<\frac{\varepsilon}{2}}}\left(\mu\left(x_{k, l}-L, t\right) \text { or } v\left(x_{k, l}-L, t\right)\right) \\
& \leq \frac{M}{h_{r s}} \mid\left\{(k, l) \in I_{r s}:\left(\mu\left(x_{k, l}-L, t\right) \text { or } v\left(x_{k, l}-L, t\right)\right) \geq \frac{\varepsilon}{2}\right\} \mid+\frac{\varepsilon}{2} .
\end{aligned}
$$

Consequently, we get

$$
\begin{aligned}
& \left\{(r, s) \in \square x \square: \frac{1}{h_{r s}} \sum_{(k, l) \in I_{r s}} \mu\left(x_{k, l}-L, t\right) \leq 1-\varepsilon \text { or } \frac{1}{h_{r s}} \sum_{(k, l) \in I_{r s}} v\left(x_{k, l}-L, t\right) \geq \varepsilon\right\} \\
& \subseteq\left\{(r, s) \in \square: \frac{1}{h_{r s}} \mid\left\{(k, l) \in I_{r s}: \mu\left(x_{k, l}-L, t\right) \leq 1-\frac{\varepsilon}{2} \text { or } v\left(x_{k, l}-L, t\right) \geq \frac{\varepsilon}{2}\right\} \mid \geq \frac{\varepsilon}{2 M}\right\} \in I .
\end{aligned}
$$

This proves the result.

iii) Follows from (i) and (ii).

Theorem 2.3. For any double lacunary sequence $\theta, I$ - double statistical convergence with respect to the intuitionistic fuzzy norm $(\mu, v)$ implies $I$ - double lacunary statistical convergence with respect to the intuitionistic fuzzy norm $(\mu, v)$ if and only if $\liminf _{r} q_{r}>1$ and $\inf _{s} q_{s}>1$. If $\liminf _{r} q_{r}=1$, and $\liminf _{s} q_{s}=1$, then there exists a 
bounded double sequence $x=\left(x_{k l}\right)$ which is $I$ - double statistically convergent but not I- double lacunary statistically convergent.

Proof. Suppose first that $\liminf _{r} q_{r}>1$ and $\liminf _{s} q_{s}>1$. Then there exists $\alpha>0$ such that $q_{r} \geq 1+\alpha$ for sufficiently large $r$ ve $q_{s} \geq 1+\alpha$ for sufficiently large $s$ which implies that

$\frac{h_{r}}{k_{r}} \geq \frac{\alpha}{\alpha+1}$.

$\frac{h_{s}}{k_{s}} \geq \frac{\alpha}{\alpha+1}$.

Since $\quad x_{k, l} \rightarrow L\left(S^{(\mu, v)}(I)_{2}\right)$, for every $\varepsilon>0, t>0$, and for sufficiently large $r, s$, we have

$$
\begin{aligned}
& \frac{1}{k_{r s}} \mid\left\{k \leq k_{r} \text { and } l \leq l_{s}: \mu\left(x_{k, l}-L, t\right) \leq 1-\varepsilon \text { or } v\left(x_{k, l}-L, t\right) \geq \varepsilon\right\} \mid \\
& \geq \frac{1}{k_{r s}} \mid\left\{(k, l) \in \mathrm{I}_{r s}: \mu\left(x_{k, l}-L, t\right) \leq 1-\varepsilon \text { or } v\left(x_{k, l}-L, t\right) \geq \varepsilon\right\} \mid \\
& \geq\left(\frac{\alpha}{\alpha+1}\right)^{2} \frac{1}{h_{r s}}\left\{\left\{(k, l) \in \mathrm{I}_{r s}: \mu\left(x_{k, l}-L, t\right) \leq 1-\varepsilon \text { or } v\left(x_{k, l}-L, t\right) \geq \varepsilon\right\} \mid \cdot\right.
\end{aligned}
$$

Then, for any $\delta>0$, we get

$$
\begin{aligned}
& \left.\left\{(r, s) \in \square \times \square: \frac{1}{h_{r s}} \mid\left\{(k, l) \in I_{r s}: \mu\left(x_{k, l}-L, t\right) \leq 1-\varepsilon \text { or } v\left(x_{k, l}-L, t\right)\right) \geq \varepsilon\right\} \mid \geq \delta\right\} \\
& \subseteq\left\{(r, s) \in \square \times \square: \frac{1}{k_{r s}} \mid\left\{k \leq k_{r} \text { and } l \leq l_{s}: \mu\left(x_{k, l}-L, t\right) \leq 1-\varepsilon \text { or } v\left(x_{k, l}-L, t\right) \geq \varepsilon\right\} \mid\right\} \\
& \geq\left(\frac{\alpha}{\alpha+1}\right)^{2} \delta \in I .
\end{aligned}
$$

This proves the sufficiency. Conversely, suppose that $\liminf _{r} q_{r}=1$ and $\liminf _{s} q_{s}=1$. We can select a subsequence $\left\{k_{r_{s}}\right\}$ and $\left\{l_{s_{j}}\right\}$ of the lacunary sequence $\theta$ such that $\frac{k_{r_{i}}}{k_{r_{i}}-1}<1+\frac{1}{i}$ and $\frac{k_{r_{i}-1}}{k_{r_{i-1} 1}}>i, \quad$ where $r_{i} \geq r_{i-1}+2$ and 
$\frac{l_{s_{j}}}{l_{s_{j}}-1}<1+\frac{1}{j}$ and $\frac{l_{s_{j}-1}}{l_{s_{j-1}}}>j$, where $s_{j} \geq s_{j-1}+2$.

Define a sequence $x=\left(x_{k, l}\right)$ by $x=\left(x_{k, l}\right)\left\{\begin{array}{lr}1, & \text { if } k \in I_{r_{i}} \text { and } l \in I_{s_{j}} \\ 0, & \text { otherwise. }\end{array}\right.$

Then, for any real $L$,

$\frac{1}{h_{r_{i} s_{j}}} \sum_{(k, l) \in I_{r^{s} j}}\left(\mu\left(x_{k, l}-L, t\right)\right.$ or $\left.v\left(x_{k, l}-L, t\right)\right)=(\mu(1-L, t)$ or $v(1-L, t))$ for $i, j=1,2, \ldots$

and

$\frac{1}{h_{r s}} \sum_{(k, l) \in I_{r s}}\left(\mu\left(x_{k, l}-L, t\right)\right.$ or $\left.v\left(x_{k, l}-L, t\right)\right)=(\mu(L, t)$ or $v(L, t))$ for $r \neq r_{i}$ and $s \neq s_{j}$.

Then it is quite clear that $x$ does not belong to $N_{\theta}^{(\mu, v)}(I)_{2}$. Since $x$ is bounded, Theorem 2.2 (iii) implies $x \nrightarrow L\left(S_{\theta}^{(\mu, v)}(I)_{2}\right)$.

Next, let $k_{r_{i}-1} \leq m \leq k_{r_{i+1}-1}$ and $l_{s_{j}-1} \leq n \leq l_{s_{j+1} .1}$. Then, from Theorem 2.2. in [7], we can write

$\frac{1}{m n} \mid\left\{k \leq m\right.$ and $l \leq n: \mu\left(x_{k, l}-L, t\right) \leq 1-\varepsilon$ or $\left.v\left(x_{k, l}-L, t\right) \geq \varepsilon\right\} \mid$

$\leq \frac{1}{m n} \sum_{k, l=1,1}^{m n}\left(\mu\left(x_{k, l}-L, t\right)\right.$ or $\left.v\left(x_{k, l}-L, t\right)\right) \leq \frac{k_{r_{j-1}} l_{s_{i-1}}+h_{r_{j} s_{i}}}{k_{r_{j}-1} l_{s_{i}-1}}<\left(\frac{2}{j i}\right)$.

Hence $\left(x_{k, l}\right)$ is $I$-double statistically convergent with respect to the intuitionistic fuzzy norm $(\mu, v)$ for any admissible ideal $I$.

\section{CONCLUDING REMARKS}

Among various developments of the theory of fuzzy sets [19] a progressive development has been made to find the fuzzy analogues of the classical set theory. In fact the fuzzy theory has become an area of active research for the last 40 years.

It has a wide range applications in the field of science and and engineering ,e.g., population dynamics, chaos control, computer programming, nonlinear dynamical systems, fuzzy physics, fuzzy topology, etc.

Recently fuzzy topology proves to be a very useful tool to deal with such situation where the use of classical theories breaks down.

In this paper, the notions of ideal double statistical convergence and ideal lacunary double statistical convergence are introduced in an intuitionistic fuzzy normed linear space, which naturally extend the notions of double statistical convergence and double lacunary statistical convergence and some important results are established. 


\section{REFERENCES}

1. H. Fast, Sur la convergence statistique, Colloquium Mathematicum 2, 241-244, 1951.

2. I.J. Schoenberg, The integrability of certain functions and related summability methods, The American Mathematical Monthly 66, 361-375, 1959.

3. J.A. Fridy, On statistical convergence, Analysis 5, 301-313, 1985.

4. T. Salat, On statistically convergent sequences of real numbers, Mathematica Slovaca 30, 139-150, 1980.

5. R. C. Buck, Generalized asymptotic density, The American Journal of Mathematics 75, 335-346, 1953.

6. J.A. Fridy, C. Orhan, Lacunary statistical convergence, Pacific Journal of Mathematics 160 ,43-51, 1993.

7. E.Savaş, R. F.Patterson, Lacunary statistical convergence of multiple sequences. Applied Mathematics Letters 19(6), 527-534, 2006.

8. S.A. Mohiuddine, M. Aiyub, Lacunary statistical convergence in random 2-normed spaces, Applied Mathematics \& Information Sciences 6(3), 581-585, 2012.

9. M. Mursaleen, S.A. Mohiuddine, On lacunary statistical convergence with respect to the intuitionistic fuzzy normed space, Journal of Computational and Applied Mathematics 233, 142-149, 2009.

10. S.A. Mohiuddine, E. Savaş, Lacunary statistically convergent double sequences in probabilistic normed spaces, Annali Dell'universita Di Ferrara, 58, 331-339, 2012.

11. G. D. Maio and L. D. R. Kocinac, Statistical convergence in topology, Topology and its Applications 156, 28-45, 2008.

12. E. Savaş, On some double lacunary sequence spaces of fuzzy numbers. Mathematical and Computational Applications 15(3), 439-448, 2010.

13. P. Das, E. Savaş, S.Kr. Ghosal, On generalizations of certain summability methods using ideals, Applied Mathematics Letters 24, 1509-1514, 2011.

14. A.R. Freedman, J.J. Sember, M. Rapnael, Some Cesaro type summability spaces, Proceedings London Mathematical Society 37, 508--520. 1978 ,

15. J. Li, Lacunary statistical convergence and inclusion properties between Lacunary methods, International Journal of Mathematics and Mathematical Sciences 23(3), 175$180,2000$.

16. E. Savaş, On $I$-asymptotically lacunary statistical equivalent sequences, Advances in Difference Equations, 2013:111. 2013.

17. E. Savaş, A note on double lacunary statistical sigma-convergence of fuzzy numbers, Soft computing, 16(4), 591-595. 2012.

18. M. Sen, P. Debnath, Lacunary statistical convergence in intuitionistic fuzzy $n$ normed linear spaces, Mathematical and Computer Modelling 54, 2978-2985, 2011.

19. L.A. Zadeh, Fuzzy sets, Inform. Control, 8, 338-353, 1965.

20. K.T. Atanassov, Intuitionistic fuzzy sets, Fuzzy Sets and Systems 20, 87-96, 1986

21. K. Atanassov, G. Pasi, R. Yager, Intuitionistic fuzzy interpretations of multi-person multicriteria decision making, in: Proceedings of 2002 First International IEEE Symposium Intelligent Systems, 1, 115-119, 2002.

22. J.H. Park, Intuitionistic fuzzy metric spaces, Chaos Solitons Fractals, 22, 10391046, 2004. 
23. R. Saadati, J.H. Park, On the intuitioistic fuzzy topologicial spaces, Chaos Solitons Fractals, 27, 331-344, 2006.

24. S. Karakus, K. Demirci, O. Duman, Statistical convergence on intuitionistic fuzzy normed spaces, Chaos Solitons Fractals 35, 763-769, 2008.

25. M. Mursaleen, S.A. Mohiuddine, H.H. Edely, On the ideal convergence of double sequences in intuitionistic fuzzy normed spaces, Computers \& Mathematics with Applications, 59, 603-611, 2010.

26. E. Savaş, Some $I$-convergent sequence spaces of fuzzy numbers defined by infinite matrix, Mathematical and Computational Applications 18(2), 84-93, 2013.

27. E. Savaş, Some double lacunary I-convergent sequence spaces of fuzzy numbers defined by Orlicz function, Journal of intelligent Fuzzy Systems, 23(5), 249-257, 2012.

28. E. Savaş, On generalized A- difference strongly summable sequence spaces defined

by ideal convergence on a real n-normed space, Journal of Inequalities and Applications, Article Number: 87 DOI: 10.1186/1029-242X-2012-87 Published: 2012.

29. E. Savaş, On some AI -convergent difference sequence spaces of fuzzy numbers defined by the sequence of Orlicz functions, Journal of Inequalities and Applications 2012, 2012:261 (6November 2012).

30. B. Schweizer, A. Sklar, Statistical metric spaces, Pacific Journal of Mathematics 10, 313-334, 1960.

31. V. Kumar, M. Mursaleen, On $(\lambda, \mu)$ - Statistical convergence of double sequences on intuitionistic fuzzy normed spaces, Filomat 25(2), 109-120, 2011.

32. Mursaleen and O. H. Edely, Statistical convergence of double sequences, Journal of Mathematical Analysis and Applications 288(1), 223-231, 2003.

33. E. Savaş and Mursaleen, On statistically convergent double sequence of Fuzzy numbers, Information Sciences, 162, 183-192, 2004.

34. P. Kostyrko, T. Salat, W. Wilczynki, I-convergence, Real Anal. Exchange 26 (2), 669-685, 2000-2001.

35. P. Kostyrko, M. Macaj, T. Salat, M. Sleziak, I-convergence and extremal I-limit points,B Mathematica Slovaca 55, 443-464, 2005.

36. B. K. Lahiri, Pratulananda Das, I and $I_{*}$-convergence in topological spaces, Mathematica Bohemica 130,153-160, 2005.

37. E. Savaş, On generalized double statistical convergence via ideals, The Fifth Saudi Science Conference, 16-18, 2012.

38. E. Savaş, P. Das and D. Sudipta: A note on strong matrix summability via ideals Applied mathematics letters, 25(4), 733-738, DOI:10.1016/j.aml.2011.10.012, 2012, 39. E.Savaş, Pratulananda Das, A generalized statistical convergence via ideals, Applied Mathematics Letters 24, 826-830, 2011.

40. E. Savaş, On convergent double sequence spaces of fuzzy numbers defined by ideal and Orlicz function, Journal of Intelligent Fuzzy Systems, 26, 1869-1877, DOI:10.3233/IFS-130866, 2014.

41. E. Savaş, On strong double matrix summability via ideals, Filomat, 26(6), 11431150, DOI 10.2298/FIL1206143S, 2012. 